\title{
Correlación entre el acortamiento de la clavícula con la función de la extremidad torácica en pacientes operados por fractura de clavícula
}

\author{
Correlation between clavicle shortening with the function of thoracic \\ extremity in patients operated by clavicle fractures \\ Pantaleón-Fierros E*
}

Hospital General Regional IMSS Villa Coapa.

RESUMEN. Introducción: La clavícula es el único hueso que une al esqueleto axial con el esqueleto apendicular; durante la función de la extremidad torácica, un acortamiento clavicular implica que haya disminución de fuerza y de función, dado que ésta actúa como palanca, ya que a mayor acortamiento menor será la fuerza producida. Factores como la multifragmentación, la pérdida ósea y la reabsorción de los bordes de los fragmentos son algunas limitantes de la restitución de la longitud clavicular. ¿Cuánto podría afectar un acortamiento de la clavícula a la función de la extremidad torácica? Material y métodos: Se realizó un estudio retrospectivo y transversal no probabilístico en pacientes con fractura clavicular que fueron tratados quirúrgicamente. El acortamiento fue medido en milímetros en la radiografía postoperatoria; después se aplicó la escala de DASH (Disabilities of the Arm, Shoulder and Hand: Discapacidad de hombro, codo y mano) para determinar el grado de la función. Resultados: Se evaluaron 12 pacientes y la edad promedio de ellos fue $37 \pm 17.7$ años; dos mujeres y 10 hombres. Fueron evaluados cinco pacientes del lado derecho y siete del lado izquierdo. Se realizó un análisis estadístico con coeficiente de correlación de Pearson. El acortamiento promedio fue de $13.32 \pm 9.9 \mathrm{~mm}$. El promedio en la escala de DASH fue de $6.0 \pm 3$. Discusión: La correlación fue moderada junto con la limitación en la función, en acortamientos $\geq 1 \mathrm{~cm}$, así como que también hubiese un significado clínico en la escala de DASH a partir de 6 puntos, 4 menos de lo que marca la literatura. Con esto se puede
ABSTRACT. Introduction: The collarbone is the only bone that joins the axial skeleton with the appendicular, during the function of the thoracic limb a shortening implies a decrease in strength and function since it acts as a lever, where the greater the shortening, the smaller will be the force produced. Factors such as multifragmentation, bone loss and reabsorption of the edges of the fragments are some limiting to the restitution of the clavicular length. How much might a shortening of the collarbone affect the function of the upper limb? Material and methods: A retrospective and non-probabilistic cross-sectional study was conducted in surgically treated clavicular fracture patients. Shortening was measured in millimeters on postoperative X-Ray, dash scale was subsequently applied to determine the degree of function. Results: 12 patients, average age $37 \pm 17.7$ years. 2 women and 10 men. 5 on the right side and 7 left side. Statistical analysis was performed with Pearson correlation coefficient. Average shortening $13.32 \pm 9.9 \mathrm{~mm}$. Average scale of DASH 6.0 \pm 3. Discussion: Moderate correlation with limitation in function in shortenings $\geq 1 \mathrm{~cm}$, as well as clinical significance on the DASH scale from 6 points, 4 less than what the literature marks. With this, it can be determined that patients managed with or without surgery, with a shortening $>1 \mathrm{~cm}$ will have a considerable limitation in the function of the shoulder and upper limb.

Nivel de evidencia: IV

* Cirujano Ortopedista. Hospital General Regional IMSS Villa Coapa, Ciudad de México.

Dirección para correspondencia:

Dr. Everardo Pantaleón Fierros

Hospital General Regional Núm. 2, IMSS,

Calzada de las Bombas Núm. 117, Col. Girasoles I, C.P. 14310, Alcaldía Coyoacán, CDMX.

E-mail: dreverpantaleon@gmail.com panta_e@hotmail.com

https://dx.doi.org/10.35366/93345

doi: $10.35366 / 93345$ 
determinar que los pacientes manejados con o sin cirugía, con un acortamiento $>1 \mathrm{~cm}$ tendrán una limitación considerable en la función del hombro y la extremidad superior.

Palabras clave: Fractura, clavícula, acortamiento, función, hombro.

\section{Introducción}

Las fracturas de clavícula suponen entre 2.6 y $4 \%$ de todas las fracturas del adulto y corresponden a $35 \%$ de las fracturas de la cintura escapular, ${ }^{1}$ de las cuales entre 70 a $80 \%$ de las fracturas de clavícula ocurre en el tercio medio. ${ }^{2}$ La edad promedio de los pacientes con fractura de clavícula es de 33 años; en cuanto a la relación hombres/mujeres se estima que $70 \%$ de las fracturas de clavícula ocurre en hombres, derivado de que la mayoría de este tipo de fracturas ocurre durante la realización de deportes de contacto o actividades de alta energía. ${ }^{3}$

Se ha llevado a cabo una mayor documentación de la incidencia de seudoartrosis o de consolidaciones en mala posición tras el tratamiento conservador de las fracturas, lo cual parece ser un tanto elevado con respecto de lo que se suponía anteriormente. De todas las fracturas del tercio medio de la clavícula, aproximadamente las dos terceras partes tienen algún tipo de consolidación viciosa; ${ }^{4}$ recientemente, se han descrito porcentajes de seudoartrosis superiores a $15 \%$ tras el tratamiento conservador, además de una pérdida funcional y pérdida de fuerza de hasta $20-25 \% .3,3,6,7,8,9,9,10$

La escala DASH es el cuestionario más empleado para la valoración global de la extremidad superior, ${ }^{11}$ permite la medición de resultados que registran objetivamente la función del miembro superior desde la perspectiva de los pacientes y se ocupa particularmente de las dificultades en las actividades recreativas o en el trabajo. Es un cuestionario de 30 ítems que evalúa síntomas y función, está dividido en tres áreas: físico (21 ítems), síntomas (seis ítems) y función social (tres ítems). Posee dos secciones opcionales que producen escalas para la participación en actividades deportivas (cuatro ítems) o de trabajo (cuatro ítems). Cada respuesta está evaluada con una escala de Linkert de 5 puntos, desde 1 (ninguna dificultad) hasta 5 (imposible de realizar). Las actividades evaluadas incluyen abrir un tarro, escribir, girar una llave, preparar comida, abrir puertas, situar objetos sobre la cabeza, etcétera. Todos los ítems se refieren a lo acontecido una semana previa a la fecha de utilización de la escala. Las respuestas se suman, a ese total se le restan 30 y luego se divide por 1.2 para obtener un valor de 0 a 100 . A mayor valor, mayor discapacidad; además, se consideran como variaciones con trascendencia clínica a aquéllas que superan los 10 puntos. $^{12}$

Es un criterio médico que, en pacientes con fractura de clavícula, ésta se ve modificada en su longitud; tradicionalmente para tomar la decisión, si es mayor a 2 cm se realizará
Keywords: Fracture, clavicle, shortening, function, shoulder.

tratamiento quirúrgico y si es menor a los $2 \mathrm{~cm}$ se realizará de manera conservadora; sin embargo, no se cuenta con la información de si el acortamiento clavicular postquirúrgico guarda una relación con el estado funcional del hombro. Es por ello que en el presente trabajo se desea conocer si existe una correlación entre dichos aspectos y si esta información pudiera ser útil para poder predecir la evolución funcional del hombro y la extremidad torácica con base en el grado de acortamiento clavicular.

Existirá una correlación de por lo menos 0.7 (moderada) entre el acortamiento clavicular radiológico con la funcionalidad del hombro en pacientes manejados quirúrgicamente por fractura del tercio medio de la clavícula.

En la literatura no se encontraron artículos que cuantificaran la relación entre el acortamiento de la clavícula fracturada intervenida quirúrgicamente con la funcionalidad del hombro, por lo que los autores, por experiencia clínica, proponen una relación potencialmente alta.

\section{Material y métodos}

Se contó con la autorización del Comité Local de Investigación y Ética en Investigación en Salud No. de registro R-2016-3702-24.

Los criterios de inclusión fueron los siguientes: expedientes de pacientes con diagnóstico de fractura del tercio medio de clavícula desde el 1 de Enero al 31 de Diciembre de 2014 y con tratamiento quirúrgico; que tuvieran entre 18 y 75 años y presentaran fracturas de clavícula recientes y no expuestas.

Los criterios de exclusión fueron: pacientes con fracturas expuestas y con manejo quirúrgico tardío o polifracturados; que no aceptaran la exploración clínica y que tuvieran una lesión neurológica del plexo braquial. También se tomaron en cuenta los antecedentes de mielopatía o radiculopatía cervical; artrosis glenohumeral y tendinopatía de mango rotador con deterioro cognitivo.

Los criterios de eliminación fueron: que no completaran la escala de DASH; que decidieran abandonar el estudio de forma voluntaria; pacientes que fallecieron por causas ajenas al padecimiento traumático y que tuvieran datos de deterioro cognitivo.

El muestreo fue no probabilístico de conveniencia. Considerando que el estudio está dirigido principalmente a establecer asociación y riesgo, el tamaño de muestra se calculó para ensayar la hipótesis de trabajo. Se utilizaron tablas para revelar las correlaciones y se consideró una alfa de 0.005 y 
Tabla 1: Datos generales de los pacientes.

\begin{tabular}{|c|c|c|c|c|c|}
\hline Paciente & Edad & Sexo & Lado fracturado & $\begin{array}{l}\text { Acortamiento radiográfico } \\
\text { postquirúrgico }(\mathrm{mm})\end{array}$ & DASH $(0-100)$ \\
\hline 1 & 72 & $\mathrm{~F}$ & Derecho & 9.8 & 2.5 \\
\hline 2 & 30 & M & Izquierdo & 19.0 & 1.6 \\
\hline 3 & 46 & M & Izquierdo & 13.3 & 11.6 \\
\hline 4 & 30 & M & Derecho & 1.2 & 5.8 \\
\hline 5 & 20 & M & Derecho & 5.0 & 4.1 \\
\hline 6 & 41 & M & Derecho & 0.0 & 5.8 \\
\hline 7 & 41 & M & Izquierdo & 16.2 & 9.1 \\
\hline 8 & 29 & M & Izquierdo & 24.8 & 5.0 \\
\hline 9 & 58 & M & Izquierdo & 15.5 & 7.5 \\
\hline 10 & 25 & $\mathrm{~F}$ & Izquierdo & 6.2 & 8.3 \\
\hline 11 & 34 & M & Derecho & 2.0 & 2.5 \\
\hline 12 & 18 & M & Izquierdo & 33.6 & 9.1 \\
\hline
\end{tabular}

una beta de 0.80 . Se encontró para una correlación de 0.70 $=11$ pacientes. ${ }^{13}$

\section{Métodos estadísticos}

Se utilizó estadística descriptiva para las variables cualitativas, con uso de porcentajes, tablas y figuras. Para las variables cuantitativas, dependiendo de la distribución, se usaron medidas de tendencia central y su correspondiente de dispersión. El análisis inferencial incluye el uso de la prueba t de Student o U de Mann-Whitney independiente para establecer las diferencias entre las variables cuantitativas de acuerdo con la distribución de los datos. La magnitud de la relación se establecerá con el coeficiente de correlación de Pearson o Spearman. Se consideró significativo todo valor de $\mathrm{p}<0.005$.

\section{Resultados}

De los pacientes revisados (12 en total), el promedio de edad fue de $37 \pm 15.7$ años y la mediana fue de 32 con rango intercuartil (RIC) 25 27, RIC 75 43.5. En relación con el sexo, los resultados fueron dos (16.6\%) mujeres y 10 (83.4\%) hombres. El lado afectado (fracturado) fue en cinco pacientes (41.7\%) el lado derecho y en siete (58.3\%) el izquierdo. El acortamiento fue de $13.32 \pm 9.9$ milímetros de la longitud total de la clavícula. El promedio en la escala de DASH fue de $6.0 \pm 3$; en mujeres fue de 5.4 y en hombres de 6.2 (Tabla 1). Del total, nueve pacientes (75\%) fueron enviados a rehabilitación.

Esto determina que, en este estudio, los pacientes con limitación en la función tuvieron un acortamiento mayor a 1 $\mathrm{cm}$, los cuales mostraron una escala de DASH mayor de 6 puntos (Tabla 2), con un valor de p de 0.07.

\section{Discusión}

En este estudio se encontró una correlación entre la limitación en la función con acortamientos mayores a un
Tabla 2: Correlación entre acortamiento y puntaje DASH.

\begin{tabular}{llccr} 
& \multicolumn{3}{c}{ Acortamiento mayor a $1 \mathrm{~cm}$} \\
\cline { 2 - 4 } & & $\begin{array}{c}\text { Menos } \\
\text { de } 1 \mathrm{~cm}\end{array}$ & $\begin{array}{c}1 \mathrm{~cm} \text { o } \\
\text { más }\end{array}$ & Total \\
\cline { 2 - 4 } $\begin{array}{l}\text { DASH puntuación } \\
\text { seis o menos }\end{array}$ & Seis o menos & 5 & 2 & 7 \\
Total & Más de seis & 1 & 4 & 5 \\
& & 6 & 6 & 12
\end{tabular}

centímetro, así como un significado clínico en la escala de DASH a partir de 6 puntos, 4 menos que lo que marca la escala. También se puede observar que, pese al manejo quirúrgico, no siempre se logra restituir la longitud de la clavícula de manera secundaria a diversos factores tanto en la propia personalidad de la fractura como en la técnica quirúrgica. Será importante lograr continuidad de este trabajo a mediano y largo plazo para poder determinar si con el paso del tiempo la limitación en la función aumenta o logra mantenerse, así como también el papel que juega la rehabilitación.

\section{Bibliografía}

1. Altamimi SA, McKee MD Canadian Orthopaedic Trauma Society. Nonoperative treatment compared with plate fixation $\mathrm{f}$ displaced midshaft clavicular fractures. Surgical technique. J Bone Joint Surg Am. 2008; 90: 1-8.

2. Postacchini F, Gumina S, De Santis P, Albo F. Epidemiology of clavicle fractures. J Shoulder Elbow Surg. 2002; 11: 452-6.

3. Pearson AM, Tosteson AN, Koval KJ, McKee MD, Cantu RV, Bell $\mathrm{JE}$, et al. Is surgery for displaced, midshaft clavicle fractures in adults cost-effective? results based on a multicenter randomized, controlled trial. J Orthop Trauma. 2010; 24(7): 426-433.

4. Hillen RJ, Burger BJ, Poll RG, de GA, Robinson CM. Malunion after midshaft clavicle fractures in adults. Acta Orthop. 2010; 81(3): 273-9.

5. Zlowodzki M, Zelle BA, Cole PA, Jeray K, McKee MD, Trauma Working Group. Treatment of acute midshaft clavicle fractures: systematic review of 2,144 fractures: on behalf of the EvidenceBased Orthopaedic. J Orthop Trauma. 2005; 19(7): 504-7.

6. Shen JW, Tong PJ, Qu HB. A three-dimensional reconstruction plate for displaced midshaft fractures of the clavicle. J Bone Joint Surg Br. 2008; 90(11): 1495-8. 
7. Nordqvist A, Redlund-Johnell I, von Scheele A, Petersson CJ. Shortening of clavicle after fracture. Incidence and clinical significance, a 5-year follow-up of 85 patients. Acta Orthop Scand. 1997; 68(4): 349-51.

8. Gossard JM. Closed treatment of displaced middle-third fractures of the clavicle gives poor results. J Bone Joint Surg Br. 1998; 80(3): 558.

9. Nowak J, Holgersson M, Larsson S. Can we predict long-term sequelae after fractures of the clavicle based on initial findings? A prospective study with nine to ten years of follow-up. J Shoulder Elbow Surg. 2004; 13(5): 479-86.

10. Postacchini R, Gumina S, Farsetti P, Postacchini F. Long-term results of conservative management of midshaft clavicle fracture. Int Orthop. 2010; 34(5): 731-6.
11. Hudak PL, Amadio PC, Bombardier C. Development of an upper Extremity outcome measure: the DASH (disabilities of the arm, Shoulder, and hand) [corrected]. The Upper Extremity Collaborative Group (UECG). Am J Ind Med. 1996; 29(6): 602-8

12. Hervas MT, Navarro MJ, Peiro S. Versión española del cuestionario DASH. Adaptación transcultural, fiabilidad, validez y sensibilidad a los cambios. Med Clin. 2006; 127(12): 441-7.

13. Browner WS, Black D, Newman TB, Hulley S. Estimación del tamaño de la muestra y de la potencia. En: Hulley SB, Cummings S. Diseño de la investigación clínica. Edición española. Baltimore, EUA: Edición Doyma; 2008.

Financiamiento: Ninguno. 